\title{
Discrepancy between sonographic and radiographic values after ultrasound-monitored treatment of developmental dysplasia of the hip
}

\author{
Sandra Utzschneider, Claudia Chita, Alexander C. Paulus, Christian Guenther, Volkmar Jansson, \\ Bernhard Heimkes
}

Department of Orthopaedic Surgery, Physical Medicine and Rehabilitation, University Hospital of Munich (LMU), Munich, Germany

Submitted: 8 March 2014

Accepted: 25 May 2014

Arch Med Sci 2016; 12, 1: 145-149

DOI: 10.5114 /aoms.2016.57590

Copyright (C) 2016 Termedia \& Banach

\section{Abstract}

Introduction: The purpose of the study was to examine the accordance between the actually used sonographic and radiographic standard values after ultrasound-monitored treatment of developmental dysplasia of the hip (DDH). Material and methods: One hundred and fifty-three (119 children) ultrasound-monitored treated hips (initial staging according to Graf: type IIc-IV) which attained normal ultrasound findings (type I according to Graf) during treatment and underwent an anteroposterior radiograph of the pelvis at the time of starting walking (mean age 18.6 months) were evaluated retrospectively.

Results: While all hips showed normal sonographic values (Graf type I), 26 (17\%) showed mild and $17(11.1 \%)$ severe dysplasia (by measuring the acetabular index) according to the radiographic Toennis classification system, and 29 (19\%) showed mild and 48 (31.4\%) severe dysplasia according to the Wiberg centre-edge angle.

Conclusions: This data show that the actually used sonographic and radiographic standard values concerning DDH do not correlate appropriately. It must be put up for discussion whether the radiographic standard values might be too strict. Further criteria must be developed to better assess the prognosis of residual dysplasia.

Key words: developmental dysplasia of the hip, hip ultrasound, Graf, Toennis classification, acetabular index.

\section{Introduction}

The importance of early diagnosis and treatment of developmental dysplasia of the hip (DDH) is well known and widely accepted. Ideally the treatment for DDH is initialized immediately after sonographic detection in the newborn period [1]. In the first postnatal year ultrasound is the method of choice in early diagnosis of DDH. Thereafter the monitoring of the dysplastic hips has to rely mainly on the interpretation of plain radiographs of the pelvis [1-3]. Another follow-up examination of dysplastic hips is recommended when children start walking.

The hypothesis was that if a child is adequately treated to Graf type I [4], the child should also have normal values in radiographs, because then the hip is considered mature.

\author{
Corresponding author: \\ Prof. Dr. med. Sandra \\ Utzschneider \\ Department \\ of Orthopaedic Surgery \\ Physical Medicine \\ and Rehabilitation \\ University Hospital \\ of Munich (LMU) \\ Campus Großhadern \\ Marchioninistr. 15 \\ 81377 Munich, Germany \\ Phone: +49 89 44-000 \\ Fax: +49 89 44-00-73934 \\ E-mail: sandra.utzschneider@ \\ med.uni-muenchen.de
}


Therefore, sonographic findings of patients treated for DDH were compared to the radiological findings at self-dependent walking age, ideally allowing for a conclusion regarding the usefulness of the reference systems currently in use.

\section{Material and methods}

Between 12/1998 and 6/2004, orthotic treatment of 523 newly diagnosed dysplastic hips was initiated at our department. Hips of Graf type Ilc and worse were considered to be eligible for treatment. Treatment was continued elsewhere in 206 children, who were consecutively lost to follow-up.

Children who were completely treated to Graf type I and who had a radiologic follow-up after reaching walking age were included in the study and reviewed retrospectively.

Exclusion criteria comprised concomitant neuromuscular, generalized metabolic, arthrogryposis-like or inflammatory hip disease, or any other associated anomaly.

Only qualitatively adequate sonograms and $X$-rays regarding recording technique, image quality, patient positioning, and complete data were accepted.

Ultrasound evaluation was performed using a 7.5 $\mathrm{MHz}$ linear transducer. The morphological findings were assigned to the categories IIc, D, IIIa/ IIIb, IV according to Graf's classification system [4].

Children with Graf type Ilc were treated with a Tübingen Hip Abduction Orthosis (Otto Bock, Duderstadt, Germany) until the sonographic achievement of Graf type I. Children with Graf type D, III and IV were treated with a Becker abduction orthosis. Hips of Graf type Ilc were evaluated every 3 weeks sonographically, hips of Graf type D, III and IV once per week. Hips of Graf type D, III and IV were treated with a Tübingen Hip Abduction Orthosis after maturing to Graf type IIC and treated further with this orthosis to Graf type I. Hips of Graf type III and IV that could not be reduced via therapy with a Becker abduction orthosis within 2 weeks underwent inpatient treatment with overhead extension. After 3 weeks of

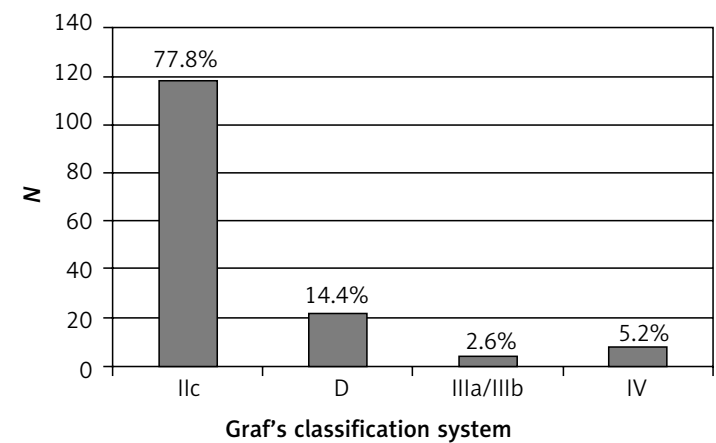

Figure 1. Sonographic types of hips, according to Graf's classification system treatment the inpatient children under overhead extension therapy underwent closed reduction and immobilisation via a spica cast for 6 weeks. After this period the cast was removed and followed by 3-month treatment with a custom made Forester-Browne orthosis.

Due to the study criteria, only children with sonographic mature hips of Graf type I after treatment were included.

The X-ray was performed after the child reached autonomous walking ability. It consisted of an anterior-posterior radiograph of the pelvis with the child in a prone position, the hips extended, patellae facing forward and the central ray aimed at the bisection of the femoral head centres. The following radiologic parameters were evaluated: measurement of the acetabular index [5], the centre-edge angle of Wiberg (CE) [6], estimation of the location of the ossific nucleus of the proximal femoral epiphysis (if present) and the position of the femoral neck.

The ultrasound findings and radiographs were independently evaluated by two experienced in vestigators.

\section{Statistical analysis}

We examined the impact of gender, positive family history and breech delivery using a $\chi^{2}$ test and a logistic regression model. A lack of amniotic fluid was not included in the analyses since too few cases were affected. $P$-values $<0.05$ were considered to be significant.

\section{Results}

Overall, 153 hips in 119 patients (101 female, 18 male) met the inclusion criteria and were evaluated in the current study. Thirty-four (28.6\%) patients were affected bilaterally. The left-right distribution was $89: 64$. Thirty-two (26.9\%) patients had a positive family history. Fifteen children were born in a breech position. Two mothers had a lack of amniotic fluid towards the end of the pregnancy.

The mean age at diagnosis and thus the start of treatment was 17.8 (1-113 min-max; SD 24.7) days. In 149 (97.4\%) hips treatment was started before the fourth month.

The sonographic types of the enclosed hips, classified according to Graf, are shown in Figure 1. A physiologic state (Graf type I) was reached at a mean age of 18.7 weeks (5-46 min-max, SD 8.9). At this time an $\alpha$ angle between 60 and $64^{\circ}$ was measured in $64(41.8 \%)$ and an $\alpha$ angle $>64^{\circ}$ in $89(58.2 \%)$ cases.

Pelvic radiographs were done at a mean age of $18.6 \pm 9.7$ months.

The $\mathrm{Al}$ according to Toennis varied from $13^{\circ}$ to $34^{\circ}$ (Figure 2). In contrast to the sonographic 
findings a residual mild or severe dysplasia (measuring the Al according to Toennis) was found in $43(28.1 \%)$ hips (Table I). These hips could be assigned to the following former initial sonographic types: whereas $26.9 \%$ of type Ilc, $27.3 \%$ of type D and $25 \%$ of type IIla/IIIb had a residual radiographic dysplasia, surprisingly $50 \%$ of the sonographic type IV had one (Figure 3). Furthermore, it is noticeable that a high proportion of the late (beginning after the third month) treated hips showed radiologically a residual dysplasia (Figure 4).

The centre-edge angle of Wiberg (CE) was $18.7^{\circ}$ (mean: $4-43^{\circ} \min -\max , \mathrm{SD} 7.5^{\circ}$ ). Thus, according to the CE angle 29 (19.0\%) mild and 48 (31.4\%) severe dysplasias were seen (Table I).

An analysis of the factors "family history", "breech presentation" and "gender" showed no impact on the radiological outcome $(p>0.05)$. Also, we found no influence of the $\alpha$-angle being above or below the threshold of $64^{\circ}$ at the time of sonographic maturity in relation to the radiographic results $(p>0.05)$.

\section{Discussion}

Although all hips were treated successfully and reached maturation according to Graf's classification system, the authors detected a high number of residual radiographic dysplasias. This indicates either a low correlation of the classification systems used, a deterioration of the hip morphology after ending the treatment or the arguability of the

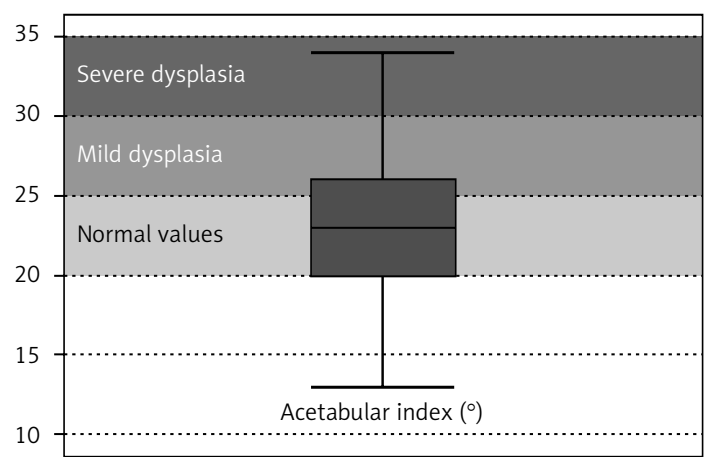

Figure 2. Distribution of the radiographic acetabular index (Al; measured in degrees; shown as a boxplot) according to Toennis [5] of the 153 hips at the age children started walking

endpoint of treatment. Also, it has to be discussed whether the radiographic standard values of the Toennis classification might be too strict. Relating to this aspect, it has to be emphasized that there is no consistent definition of DDH radiographically in the current literature. The Toennis standard values are widely used to classify residual dysplasia $[3,7,8]$. Using this classification, $28.1 \%$ of our patients showed radiographic residual dysplasia after completion of ultrasound monitored treatment. None of these patients was scheduled for operative treatment due to these findings, but the authors recommend a subsequent radiographic evaluation after two years before indicating a surgical procedure. This algorithm is feasible because

Table I. Rate of mild and severe dysplasia, measured as the acetabular index (Al) according to Toennis [5], and the centre-edge angle (CE) of Wiberg [6]

\begin{tabular}{|lccc|}
\hline Rate of residual dysplasia & None & Mild & Severe \\
\hline Acetabular index (Al) according to Toennis & 110 & 26 & 17 \\
\cline { 2 - 4 } & $71.9 \%$ & $17.0 \%$ & $11.1 \%$ \\
\hline Centre edge angle & 76 & 29 & 48 \\
\cline { 2 - 4 } & $49.7 \%$ & $19.0 \%$ & $31.4 \%$ \\
\hline
\end{tabular}

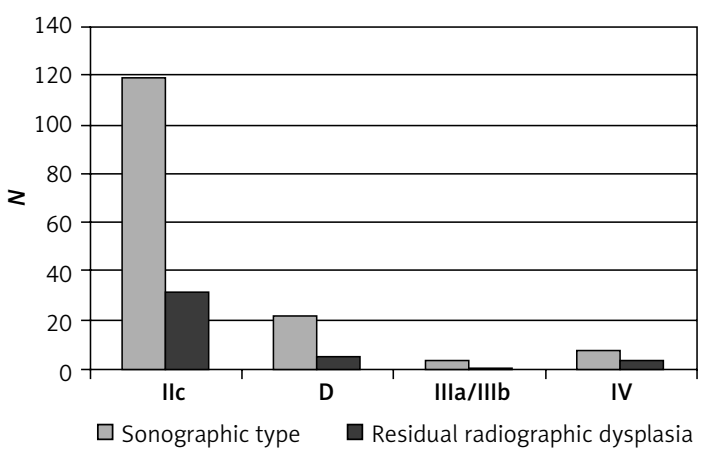

Figure 3. Contrasting juxtaposition of the former sonographic hip types according to Graf's classification system and residual radiographic dysplasia, measured as acetabular index

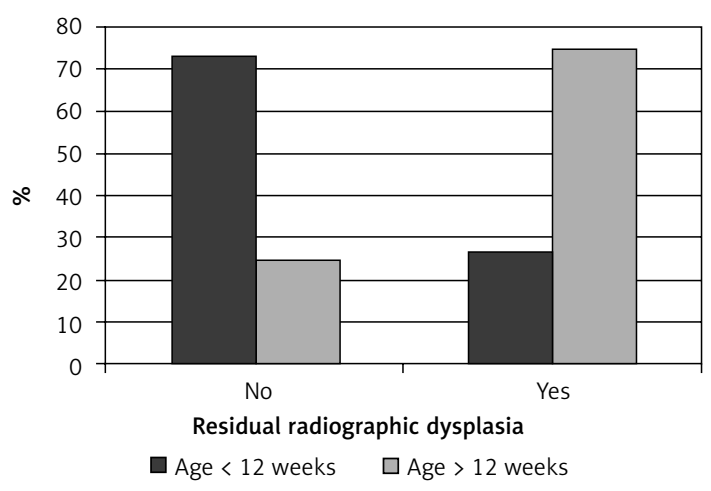

Figure 4. Illustration of the rate of residual radiographic dysplasia, measured as the acetabular index (Al), according to the beginning of treatment 
it is known that clinically stable and radiologically well-centred hips with an increased age-related acetabular index angle tend to improve spontaneously [9]. However, for these cases, clinical and radiologic follow-up is required [9].

In addition, there is some incidence of occult dysplasia of the contralateral hip in children with unilateral hip dysplasia. For this reason, it is indispensable to control these patients until skeletal maturity [10].

A large discrepancy between several radiographic definitions of residual dysplasia exists in the literature $[3,7,11,12]$. Whereas some authors define residual dysplasia as an $\mathrm{Al}$ of more than $30^{\circ}$, others define it as more than 2 SD above the mean $\mathrm{Al}$ according to the respective age $[3,7,11$, 12]. However, in our opinion, early surgical procedures have to be avoided because most of the affected hips showing residual dysplasia on the radiograph resolve in the following years $[8,13]$.

Dornacher et al. suggest delaying the first radiograph until 2 years of age [1]. They found a very high rate of residual dysplasia (62.2\%) according to the Toennis classification when children started walking. In their opinion these results reflected the delay of development of the acetabulum [1]. In our opinion this strategy should be handled with caution since some studies showed that both deterioration to subluxation or dislocation as well as spontaneous amelioration are possible without treatment [14-17].

The authors could not detect any relation between endogenous factors, such as positive medical history in the family or breech delivery, and the incidence of residual dysplasia. These findings correspond well with the clinical experience that the development of dysplastic hip cannot be predicted adequately [8].

Furthermore, a correlation between the residual dysplasia and the initial severity of DDH was not seen. This finding corresponds well with the common literature $[1,3]$.

In contrast to the literature [1,3], in the current study initial "type IV" hips according to Graf's system showed a higher tendency to retain a residual radiographic dysplasia than milder sonographic types of dysplasia. But this phenomenon must be confirmed by larger case series of "type IV" hips.

In addition, it would be interesting to investigate in another study whether the speed of normalization of the $\alpha$ angle in ultrasound corresponds with the severity of remaining radiological dysplasia, which might allow the estimation of the risk of persisting dysplasia already at an early age.

Furthermore, in the current study the authors pointed out that an early treatment (age $<12$ weeks) could play a role in avoiding residual radiographic dysplasia. But also at this point larger case series have to be awaited.
Beyond that, there are several limitations to the study. First of all, a good deal of the patients in the initial sample were lost to follow-up. Secondly, the follow-up of the involved patients ends at the time when children start walking. It would be extremely interesting to follow these patients to skeletal maturity. Perhaps then some factors could be extracted to predict the long-term outcome of the patients. Third, to the knowledge of the authors, no radiographic classification system exists to evaluate the hips at the time children start walking, which would allow for an adequate prediction of the developmental stage of dysplastic hips. In respect of the findings in the current study, there is a need for revision of the reference ranges, but it is unclear which radiographic definition properly describes a dysplastic hip.

In conclusion, there is a considerable discrepancy between the sonographic findings considered as adequately matured, initially dysplastic hips which lead to the ending of treatment and the radiological findings at walking age. This indicates that the sonographic and radiological reference values should be subjected to a critical re-evaluation. This raises the need for safe division of these hips by standardized criteria with high reliability. Further radiological development of the monitored hips up to skeletal maturity could help to identify factors predicting the probable development of $\mathrm{DDH}$.

\section{Conflict of interest}

The authors declare no conflict of interest.

\section{References}

1. Dornacher D, Cakir B, Reichel H, Nelitz M. Early radiological outcome of ultrasound monitoring in infants with developmental dysplasia of the hips. J Pediatr Orthop B 2010; 19: 27-31.

2. Tonnis D. Normal values of the hip joint for the evaluation of X-rays in children and adults. Clin Orthop Relat Res 1976; 119: 39-47.

3. Sucato DJ, Johnston CE 2nd, Birch JG, Herring JA, Mack P. Outcome of ultrasonographic hip abnormalities in clinically stable hips. J Pediatr Orthop 1999; 19: 754-9.

4. Graf R. Classification of hip joint dysplasia by means of sonography. Arch Orthop Trauma Surg 1984; 102: 248-55.

5. Tonnis D, Brunken D. [Differentiation of normal and pathological acetabular roof angle in the diagnosis of hip dysplasia. Evaluation of 2294 acetabular roof angles of hip joints in children]. Archiv fur Orthopadische und Unfall-Chirurgie 1968; 64: 197-228.

6. Wiberg G. Studies on dysplastic acetabula and congenital subluxation of the hip joint. Acta Chir Scand 1939; 83 (Suppl. 58): 7.

7. Gwynne Jones DP, Dunbar JD, Theis JC. Late presenting dislocation of sonographically stable hips. J Pediatr Orthop B 2006; 15: 257-61.

8. Cashman JP, Round J, Taylor G, Clarke NM. The natural history of developmental dysplasia of the hip after early supervised treatment in the Pavlik harness. A prospec- 
tive, longitudinal follow-up. J Bone Joint Surg Br 2002; 84: 418-25.

9. Mladenov K, Dora C, Wicart P, Seringe R. Natural history of hips with borderline acetabular index and acetabular dysplasia in infants. J Pediatr Orthop 2002; 22: 607-12.

10. Song FS, McCarthy JJ, MacEwen GD, Fuchs KE, Dulka SE. The incidence of occult dysplasia of the contralateral hip in children with unilateral hip dysplasia. J Pediatr Orthop 2008; 28: 173-6.

11. Nakamura J, Kamegaya M, Saisu T, Someya M, Koizumi W, Moriya $\mathrm{H}$. Treatment for developmental dysplasia of the hip using the Pavlik harness: long-term results. J Bone Joint Surg Br 2007; 89: 230-5.

12. Albinana J, Dolan LA, Spratt KF, Morcuende J, Meyer MD, Weinstein SL. Acetabular dysplasia after treatment for developmental dysplasia of the hip. Implications for secondary procedures. J Bone Joint Surgery Br 2004; 86: 876-86.

13. Taylor GR, Clarke NM. Monitoring the treatment of developmental dysplasia of the hip with the Pavlik harness. The role of ultrasound. J Bone Joint Surgery $\mathrm{Br}$ 1997; 79: 719-23.

14. Coleman SS. Congenital dysplasia of the hip in the Navajo infant. Clin Orthop Relat Res 1968; 56: 179-93.

15. Pratt WB, Freiberger RH, Arnold WD. Untreated congenital hip dysplasia in the Navajo. Clin Orthop Relat Res 1982; 162: 69-77.

16. Schwend RM, Pratt WB, Fultz J. Untreated acetabular dysplasia of the hip in the Navajo. A 34 year case series follow-up. Clin Orthop Relat Res 1999; 364: 108-16.

17. Weinstein SL. Natural history of congenital hip dislocation $(\mathrm{CDH})$ and hip dysplasia. Clin Orthop Relat Res 1987; 225: 62-76. 